\title{
Hölder Continuous Regularity of Stochastic Convolutions with Distributed Delay
}

\section{Kai Liu ${ }^{1}$}

Received: 16 September 2019 / Accepted: 24 January 2021 / Published online: 22 February 2021 (C) Crown 2021

\begin{abstract}
In this work, we consider the Hölder continuous regularity of stochastic convolutions for a class of linear stochastic retarded functional differential equations with distributed delay in Hilbert spaces. By focusing on distributed delays, we first establish some more delicate estimates for fundamental solutions than those given in Liu (Discrete Contin. Dyn. Syst. Ser. B 25(4), 1279-1298, 2020). Then we apply these estimates to stochastic convolutions incurred by distributed delay to study their regularity property. Last, we present some easilyverified results by considering the regularity of a class of systems whose delay operators have the same order derivatives as those in instantaneous ones.
\end{abstract}

Keywords Regularity property · Fundamental solution · Stochastic convolution

Mathematics Subject Classification (2010) 60H15 · 60G15 · 60H05

\section{Introduction}

To motivate this work, let $a:[-r, 0] \rightarrow \mathbb{R}, r>0$, be some appropriate function and $h_{0}: \mathbb{R} \rightarrow \mathbb{R}$ be a monotonically continuous function which satisfies the following relations (see, e.g., Coleman and Gurtin [1] and Nunziato [7])

$$
\begin{aligned}
x \cdot h_{0}(x) & \geq \gamma_{1}|x|^{p}+\alpha_{1}, \quad \forall x \in \mathbb{R}, \\
\left|h_{0}(x)\right| & \leq \gamma_{2}|x|^{p-1}+\alpha_{2}, \quad \forall x \in \mathbb{R},
\end{aligned}
$$

where $p \geq 2, \gamma_{i}>0$ and $\alpha_{i} \in \mathbb{R}$ for $i=1,2$. The non-Fourier heat conduction model with memory in the conductor $(0, \pi) \subset \mathbb{R}$ may start from the constitutive equation

$$
q(t, x)=-h_{0}(\partial y(t, x) / \partial x)-\int_{-r}^{0} a(\theta) \frac{\partial y(t+\theta, x)}{\partial x} d \theta, \quad t \geq 0, \quad x \in(0, \pi),
$$

Kai Liu

k.liu@liverpool.ac.uk

1 Department of Mathematical Sciences, School of Physical Sciences, The University of Liverpool, Peach Street, Liverpool, L69 7ZL, UK 
and the energy conservative equation without exterior energy sources

$$
d_{t} p(t, x)+\frac{\partial q(t, x)}{\partial x} d t=0, \quad t \geq 0, \quad x \in(0, \pi),
$$

where $y$ denotes the temperature, $q$ is the heat flux and $p$ is the internal energy which can be taken, in most situations, as the form: $p(t, x)=\kappa y(t, x), \kappa>0$. In practice, the assumption of zero exterior energy source is artificial, and a more realistic model is that the null exterior energy source is perturbed by a noise, for example, a white noise random field $b(x) \dot{w}(t, x)$, $b \in L^{2}(0, \pi)$. In other words, Eq. 1.2 is replaced at present by the following equation

$$
d_{t} p(t, x)+\frac{\partial q(t, x)}{\partial x} d t=b(x) d w(t, x), \quad t \geq 0, \quad x \in(0, \pi) .
$$

Then, by substituting Eq. 1.3 into 1.1, one can obtain, if $\kappa=1$, an equation of the form

$$
\left\{\begin{array}{l}
d_{t} y(t, x)=\frac{\partial h_{0}(\partial y(t, x) / \partial x)}{\partial x} d t+\int_{-r}^{0} a(\theta) \frac{\partial^{2} y(t+\theta, x)}{\partial x^{2}} d \theta d t+b(x) d w(t, x), \quad t \geq 0, \\
y(0, \cdot)=\phi_{0}(\cdot) \in L^{2}(0, \pi), \quad y(\theta, \cdot)=\phi_{1}(\theta, \cdot) \in W_{0}^{1, p}(0, \pi), \theta \in[-r, 0], \\
y(t, 0)=y(t, \pi)=0, \quad t \in(0, \infty) .
\end{array}\right.
$$

Let $A=d^{2} / d x^{2}, H=L^{2}(0, \pi), V=H_{0}^{1}(0, \pi), U=W_{0}^{1, p}(0, \pi), B u=b u, u \in H$, $W(t)=w(t, \cdot)$ and $h(u)=\frac{d h_{0}(d u(x) / d x)}{d x}$ for any $u \in W_{0}^{1, p}(0, \pi)$. We thus have a stochastic differential equation with distributed delay in $H$,

$$
\left\{\begin{array}{l}
d y(t)=\left(h(y(t))+\int_{-r}^{0} a(\theta) A y(t+\theta) d \theta\right) d t+B d W(t), \quad t \geq 0, \\
y(0)=\phi_{0}, \quad y(\theta)=\phi_{1}(\theta), \quad \theta \in[-r, 0],
\end{array}\right.
$$

where $U \subset H \subset U^{*}$ and $h$ is a continuous monotone operator from $U$ to $U^{*}$ such that

$$
\begin{aligned}
\langle u, h(u)\rangle_{U, U^{*}} & \geq \gamma_{1}\|u\|_{U}^{p}+\alpha_{1}, \quad \forall u \in U, \\
\|h(u)\|_{U^{*}} & \leq \gamma_{2}\|u\|_{U}^{p-1}+\alpha_{2}, \quad \forall u \in U,
\end{aligned}
$$

and $p \geq 2, \gamma_{i}>0, \alpha_{i} \in \mathbb{R}$ for $i=1,2$. In particular, if $h_{0}(x)=x, p=2$, then $h(u)=A u$, $V=U$ and the Eq. 1.5 reduces to

$$
\left\{\begin{array}{l}
d y(t)=\left(A y(t)+\int_{-r}^{0} a(\theta) A y(t+\theta) d \theta\right) d t+B d W(t), \quad t \geq 0, \\
y(0)=\phi_{0}, \quad y_{0}=\phi_{1} .
\end{array}\right.
$$

The aim of this work is to investigate the regularity property of such stochastic systems as Eq. 1.6.

The organization of this work is as follows. In Section 2, we first introduce a deterministic linear retarded functional differential equation associated in our formulation of stochastic systems. We review the useful variation of constants formula for the equations under consideration by means of a method of fundamental solutions. Also, we show some estimates of fundamental solutions which will play an important role in the subsequent development. In Sections 3 and 4, we give the detailed proofs of the key result, i.e., Theorem 2.1, by following Prüss's method of constructing some resolvent operators for the integrodifferential equations of Volterra type. By employing the main results, we establish in Section 5 the desired regularity property for stochastic convolutions. Last, we focus on some particular cases such as $A=A_{1}$ in Eq. 5.2, which allow us in Section 6 to deal with a class of systems 
with delays in the highest-order derivatives and in Section 7 to consider the continuity of stochastic convolutions not in a Hilbert space, but in a smaller Banach space of this space.

Finally, a few words about the notation are in order: generic positive constants will be denoted by $C$; we shall use the shorthand notation $a \preceq b$ to denote $a \leq C b$. If the constant $C$ depends on a parameter $p$, we may write $C_{p}$ and $a \preceq_{p} b$.

\section{Fundamental Solution}

Let $H$ be a separable Hilbert space equipped with the norm $\|\cdot\|_{H}$ and inner product $\langle\cdot, \cdot\rangle_{H}$, respectively. Also, we denote by $\mathscr{L}(H)$ the space of all bounded linear operators in $H$ and by $\|\cdot\|$ the usual operator norm in $\mathscr{L}(H)$. We are concerned with the following linear retarded functional differential equation in $H$,

$$
\left\{\begin{array}{l}
d y(t)=A y(t) d t+\int_{-r}^{0} a(\theta) A_{1} y(t+\theta) d \theta d t+f(t) d t, \quad t \geq 0, \\
y(0)=\phi_{0}, \quad y(\theta)=\phi_{1}(\theta), \quad \theta \in[-r, 0], \quad \phi=\left(\phi_{0}, \phi_{1}\right),
\end{array}\right.
$$

where $r>0$ is some constant incurring the system delay, $a(\cdot) \in L^{2}([-r, 0], \mathbb{R})$ and $\phi=$ $\left(\phi_{0}, \phi_{1}\right)$ is an appropriate initial datum. Here $A: \mathscr{D}(A) \subset H \rightarrow H$ is the infinitesimal generator of an analytic semigroup $e^{t A}, t \geq 0, A_{1}$ is a closed linear operator with domain $\mathscr{D}\left(A_{1}\right) \supset \mathscr{D}(A)$ and $f$ is some continuous function with values in $H$. For simplicity, we assume in this work that the $C_{0}$-semigroup $e^{t A}$ is negative type, i.e., there exist constants $M \geq 1$ and $\mu>0$ such that

$$
\left\|e^{t A}\right\| \leq M e^{-\mu t}, \quad\left\|A e^{t A}\right\| \leq M / t \quad \text { for all } \quad t>0,
$$

and for $\gamma \in(0,1)$, there exists a constant $M_{\gamma}>0$ such that

$$
\left\|(-A)^{\gamma} e^{t A}\right\| \leq M_{\gamma} / t^{\gamma} \quad \text { for all } \quad t>0,
$$

where $(-A)^{\gamma}$ is the standard fractional power of operator $A$.

Equations of the type (2.1) were investigated by many researchers such as Di Blasio, Kunisch and Sinestrari [3] and the fundamental solution to Eq. 2.1 was introduced by Jeong, Nakagiri and Tanabe [5]. In particular, it is known that the fundamental solution $G(\cdot)$ : $\mathbb{R} \rightarrow \mathscr{L}(H)$ to Eq. 2.1 is an operator-valued function which is strongly continuous in $H$ and satisfies the integral equation

$$
G(t)= \begin{cases}e^{t A}+\int_{0}^{t} \int_{-r}^{0} a(\theta) e^{(t-s) A} A_{1} G(s+\theta) d \theta d s, & t \geq 0, \\ \mathrm{O}, & t<0,\end{cases}
$$

where $\mathrm{O}$ is the null operator in $H$. The fundamental solution $G$ enables us to solve the initial value problem (2.1). In fact, it may be shown that under some reasonable conditions on $f$ and initial datum $\phi=\left(\phi_{0}, \phi_{1}\right)$, the unique mild solution $y$ to Eq. 2.1 is represented as

$$
y(t)=G(t) \phi_{0}+\int_{-r}^{0} U_{t}(\theta) \phi_{1}(\theta) d \theta+\int_{0}^{t} G(t-s) f(s) d s, \quad t \geq 0,
$$

where

$$
U_{t}(\theta)=\int_{-r}^{\theta} a(\tau) G(t-\theta+\tau) A_{1} d \tau, \quad \theta \in[-r, 0],
$$

with the initial condition $y(0)=\phi_{0}$ and $y(\theta)=\phi_{1}(\theta), \theta \in[-r, 0)$. This is a time delay version of the usual variation of constants formula without memory. 
In order to apply Eq. 2.5 to such equations as Eq. 1.6 to consider their regularity property of solutions, we need establish some inequalities in association with $G(\cdot)$. To this end, we introduce the following conditions:

$$
\begin{aligned}
& a(\cdot) \in L^{\infty}([-r, 0], \mathbb{R}) \text { and } \\
& \qquad \mathscr{D}\left((-A)^{\nu}\right) \subset \mathscr{D}\left(A_{1}\right) \quad \text { for some } \quad v \in(0,1) .
\end{aligned}
$$

(H2) $a(\cdot)$ is Hölder continuous, i.e., there exist constants $M>0$ and $\rho \in(0,1)$ such that

$$
|a(\tau)-a(\theta)| \leq M(\tau-\theta)^{\rho} \quad \text { for any } \quad-r \leq \theta<\tau \leq 0,
$$

and $A_{1} e^{t A}=e^{t A} A_{1}$ for each $t>0$ or both $A$ and $A_{1}$ are self-adjoint.

Theorem 2.1 For the fundamental solution $G(\cdot)$ to Eq. 2.4,

(a) assume that condition (H1) or (H2) holds, then for any $\gamma \in[v, 1)$ or $\gamma \in(0,1)$,

$$
\left\|(-A)^{\gamma} G(t)\right\| \leq \frac{C_{n, \gamma}}{t^{\gamma}} \quad \text { for all } \quad 0<t \leq n r,
$$

where $C_{n, \gamma}>0, n \in \mathbb{N}:=\{1,2, \cdots\}$, are constants depending on $n$ and $\gamma$;

(b) assume that condition (H1) or (H2) holds, then for any $\gamma \in[v, 1)$ or $\gamma \in(0,1)$ and $0<\beta<1-\gamma$

$$
\left\|(-A)^{\gamma}(G(t)-G(s))\right\| \leq C_{1, \beta, \gamma} \frac{(t-s)^{\beta}}{s^{\beta+\gamma}} \quad \text { for all } \quad 0<s<t \leq r
$$

and

$$
\left\|(-A)^{\gamma}(G(t)-G(s))\right\| \leq C_{n, \beta, \gamma} \frac{(t-s)^{\beta}}{(s-r)^{\beta}} \quad \text { for all } \quad r<s<t \leq n r,
$$

where $C_{n, \beta, \gamma}>0, n \in \mathbb{N}$, are constants depending on $n, \beta$ and $\gamma$.

Remark 2.1 In [6], it was shown that under the condition (H1), the fundamental solution $G(\cdot)$ to Eq. 2.4 satisfies:

(a) for any $\gamma \in[v, 1)$,

$$
\left\|(-A)^{\gamma} G(t)\right\| \leq \frac{C_{n, \gamma}}{(t-n r)^{\gamma}} \quad \text { for all } \quad t \in(n r,(n+1) r],
$$

where $C_{n, \gamma}>0, n \in \mathbb{N}_{0}:=\{0,1, \cdots\}$, are constants depending on $n$ and $\gamma$;

(b) for any $\gamma \in[v, 1)$ and $0<\beta<1-\gamma$,

$$
\left\|(-A)^{\gamma}(G(t)-G(s))\right\| \leq C_{n, \beta, \gamma} \frac{(t-s)^{\beta}}{(s-n r)^{\beta+\gamma}} \quad \text { for all } \quad n r<s<t \leq(n+1) r,
$$

where $C_{n, \beta, \gamma}>0, n \in \mathbb{N}$, are constants depending on $n, \beta$ and $\gamma$.

In other words, Theorem 2.1 here improves the results (2.11), (2.12) in [6] for systems with distributed delay.

Remark 2.2 In this work, we are mainly interested in unbounded operator $A_{1}$. If $A_{1}$ is bounded, Eq. 2.6 is clearly valid for $v=0$ and vise versa, and then we actually come to a trivial case. In this situation, all the results in Theorem 2.1 remain true for $\gamma \in(0,1)$ and their proofs can be significantly simplified in comparison with those in the following sections. 


\section{Proof of Theorem 2.1 (a)}

First, let us denote by $|\cdot|_{\infty}$ the essential least upper bound norm in $L^{\infty}([-r, 0], \mathbb{R})$, i.e.,

$$
|f|_{\infty}:=\operatorname{ess} \sup _{\theta \in[-r, 0]}|f(\theta)| \quad \text { for any } \quad f \in L^{\infty}([-r, 0], \mathbb{R}) .
$$

It is immediate that $|a|_{\infty}=\max _{\theta \in[-r, 0]}|a(\theta)|$ if $a(\cdot)$ is Hölder continuous. For $\gamma \in(0,1)$, we define

$$
\Gamma(t)=\int_{0}^{t}(-A)^{\gamma} e^{(t-s) A} a(-s) d s, \quad t \in[0, r] .
$$

The proof of the following proposition is referred to Liu [6].

Proposition 3.1 The mapping $\Gamma(\cdot):[0, r] \rightarrow \mathscr{L}(H)$ is uniformly bounded and for any $0<\beta<1-\gamma$, there exists a number $C_{\beta, \gamma}>0$ such that

$$
\|\Gamma(t)-\Gamma(s)\| \leq C_{\beta, \gamma}(t-s)^{\beta}, \quad 0 \leq s<t \leq r,
$$

i.e., $\Gamma$ is Hölder continuous on $[0, r]$ with order $\beta \in(0,1-\gamma)$.

To show Theorem 2.1 (a), we intend to develop an induction scheme. We first consider the case that $n=0$ and set

$$
V(t)=(-A)^{\gamma}\left(G(t)-e^{t A}\right), \quad t \in[0, r] .
$$

Then, the integral equation to be satisfied by $V(t)$ is

$$
V(t)=V_{0}(t)+\int_{0}^{t} \Gamma(t-s) A_{1}(-A)^{-\gamma} V(s) d s, \quad t \in[0, r],
$$

where

$$
\begin{aligned}
V_{0}(t) & =\int_{0}^{t} \Gamma(t-s) A_{1} e^{s A} d s \\
& =\int_{0}^{t}(\Gamma(t-s)-\Gamma(t)) A_{1} e^{s A} d s+\Gamma(t) A_{1} A^{-1}\left(e^{t A}-I\right), \quad t \in[0, r] .
\end{aligned}
$$

Since $\mathscr{D}(A) \subset \mathscr{D}\left(A_{1}\right), A_{1} A^{-1}$ is defined on all of $H$ and by the well-known closed graph theorem we have $A_{1} A^{-1} \in \mathscr{L}(H)$. Then for any $0<\beta<1-\gamma$, it follows by virtue of Proposition 3.1 and Eq. 2.2 that

$$
\begin{aligned}
\left\|V_{0}(t)\right\| & \leq \int_{0}^{t}\left\|(\Gamma(t-s)-\Gamma(t)) A_{1} A^{-1} A e^{s A}\right\| d s+\|\Gamma(t)\|\left\|A_{1} A^{-1}\right\|\left(\left\|e^{t A}\right\|+1\right) \\
& \leq \int_{0}^{t} C_{\beta, \gamma} M\left\|A_{1} A^{-1}\right\| s^{\beta-1} d s+\|\Gamma\|_{\infty}\left\|A_{1} A^{-1}\right\|(M+1) \\
& \leq \frac{C_{\beta, \gamma} M\left\|A_{1} A^{-1}\right\|}{\beta} r^{\beta}+\|\Gamma\|_{\infty}\left\|A_{1} A^{-1}\right\|(M+1), \quad t \in[0, r] .
\end{aligned}
$$

Hence, $V_{0}(\cdot)$ is uniformly bounded on $[0, r]$.

If (H1) holds, we have $A_{1}(-A)^{-v} \in \mathscr{L}(H)$ and

$$
\left\|A_{1}(-A)^{-\gamma}\right\| \leq\left\|A_{1}(-A)^{-v}\right\| \cdot\left\|(-A)^{-(\gamma-v)}\right\|<\infty .
$$

Hence, by virtue of the well-known Gronwall lemma, Eqs. 3.4 and 3.5, it is easy to have

$$
v_{0}:=\sup _{0 \leq t \leq r}\|V(t)\|<\infty .
$$


Now assume that condition (H2) holds, then we have by the relation $A_{1} A^{-1} \in \mathscr{L}(H)$ that

$$
\left\|e^{t A} A_{1}\right\|=\left\|A_{1} A^{-1}\right\| \cdot\left\|A e^{t A}\right\| \leq C_{1} t^{-1} \quad \text { for some } \quad C_{1}>0 \quad \text { and all } \quad t>0 .
$$

Also, it is easy to have that for any $u \in \mathscr{D}(A)$ and $t>0$,

$$
\left\|(-A) e^{t A} A_{1} u\right\|=\left\|A e^{t A} A_{1} A^{-1} A u\right\| \leq C_{2} t^{-1}\left\|A_{1} A^{-1}\right\| \cdot\|A u\|, \quad C_{2}>0,
$$

which, by virtue of Theorem 2.3.4, pp. 45-46, [9], immediately implies that for any $x \in H$,

$$
\begin{aligned}
\left\|(-A)^{\gamma} e^{t A} A_{1}(-A)^{-\gamma} x\right\|_{H} & \leq C \cdot\left\|A_{1} A^{-1}\right\| t^{-(1-\gamma)}\left\|(-A)^{\gamma}(-A)^{-\gamma} x\right\|_{H} \\
& =C \cdot\left\|A_{1} A^{-1}\right\| t^{-(1-\gamma)}\|x\|_{H}, \quad t>0,
\end{aligned}
$$

for some constant $C>0$. Hence, by virtue of the Gronwall lemma, Eqs. 3.2, 3.4 and 3.6, we have

$$
\begin{aligned}
v_{0} & \leq \sup _{0 \leq t \leq r}\left\|V_{0}(t)\right\| \exp \left(\int_{0}^{t}\left\|\Gamma(t-s) A_{1}(-A)^{-\gamma}\right\| d s\right) \\
& \leq \sup _{0 \leq t \leq r}\left\|V_{0}(t)\right\| \cdot \exp \left(|a|_{\infty} \int_{0}^{r} \int_{0}^{s}\left\|(-A)^{\gamma} e^{(s-u) A_{1}} A_{1}(-A)^{-\gamma}\right\| d u d s\right) \\
& \leq \sup _{0 \leq t \leq r}\left\|V_{0}(t)\right\| \cdot \exp \left(|a|_{\infty} C\left\|A_{1} A^{-1}\right\| \int_{0}^{r} \int_{0}^{s} \frac{1}{(s-u)^{1-\gamma}} d u d s\right) \\
& =\sup _{0 \leq t \leq r}\left\|V_{0}(t)\right\| \exp \left(\frac{|a|_{\infty} C\left\|A_{1} A^{-1}\right\| r^{1+\gamma}}{\gamma(1+\gamma)}\right)<\infty .
\end{aligned}
$$

Note that

$$
(-A)^{\gamma} G(t)=(-A)^{\gamma} e^{t A}+V(t), \quad t \in[0, r] .
$$

Hence, for $t \in(0, r]$ we have

$\left\|(-A)^{\gamma} G(t)\right\| \leq\left\|(-A)^{\gamma} e^{t A}\right\|+\|V(t)\| \leq M_{\gamma} / t^{\gamma}+v_{0} \leq\left(M_{\gamma}+v_{0} r^{\gamma}\right) / t^{\gamma}, \quad M_{\gamma}>0$, which is Eq. 2.8 with $n=1$.

Now suppose the fundamental solution $G(\cdot)$ satisfies all the estimates in Theorem 2.1 (a) on the intervals $[0, n r]$. Then in the interval $(n r,(n+1) r]$, the integral equation to be satisfied by

$$
V(t)=(-A)^{\gamma} G(t), \quad t \in(n r,(n+1) r],
$$

is

$$
V(t)=V_{0}(t)+\int_{n r}^{t} \Gamma(t-u) A_{1}(-A)^{-\gamma} V(u) d u,
$$

where

$$
\begin{aligned}
V_{0}(t) & =(-A)^{\gamma} e^{t A}+\int_{0}^{t-r} e^{(t-r-u) A} \Gamma(r) A_{1} G(u) d u+\int_{t-r}^{n r} \Gamma(t-u) A_{1} G(u) d u \\
& =: I_{1}(t)+I_{2}(t)+I_{3}(t) .
\end{aligned}
$$

If condition (H1) holds, it is known by Liu [6] that Eq. 2.8 is true on $[0,(n+1) r]$. Now assume that $(\mathrm{H} 2)$ holds and we estimate each term on the right hand side of Eq. 3.11. First, note that

$$
\left\|I_{1}(t)\right\| \leq \frac{M_{\gamma}}{t^{\gamma}}, \quad t \in(n r,(n+1) r] .
$$

It is known by Theorem 1 in Tanabe [8] that for $i=0,1, A_{0}=A$,

$$
\left\|A_{i} G(t)\right\| \leq \frac{C_{j}^{\prime}}{t-j r}, \quad\left\|\int_{s}^{t} A_{i} G(u) d u\right\| \leq C_{j}^{\prime}, \quad j r \leq s<t \leq(j+1) r,
$$


for some $C_{j}^{\prime}>0, j \in\{0,1,2, \cdots\}$. To estimate $I_{2}(t)$, we first note that

$$
\left\|e^{t A}-e^{s A}\right\| \leq C_{\alpha}(t-s)^{\alpha} \cdot s^{-\alpha}, \quad C_{\alpha}>0
$$

for any $0<s<t<\infty$ and $\alpha \in(0,1]$ (cf. (4.23) in [6]). Then we re-write $I_{2}(t)$ as

$$
\begin{aligned}
& \int_{0}^{t-r} e^{(t-u-r) A} \Gamma(r) A_{1} G(u) d u \\
& =\sum_{j=0}^{n-2} \int_{j r}^{(j+1) r} e^{(t-u-r) A} \Gamma(r) A_{1} G(u) d u+\int_{(n-1) r}^{t-r} e^{(t-u-r) A} \Gamma(r) A_{1} G(u) d u .
\end{aligned}
$$

For $j=0,1, \cdots, n-2$ and $t \in(n r,(n+1) r]$, we have by Eqs. 3.13 and 3.14 that

$$
\begin{aligned}
\left\|\int_{j r}^{(j+1) r} e^{(t-u-r) A} \Gamma(r) A_{1} G(u) d u\right\| \\
\quad \leq \quad \int_{j r}^{(j+1) r}\left\|e^{(t-u-r) A}-e^{(t-j r-r) A}\right\| \cdot\|\Gamma(r)\| \cdot\left\|A_{1} G(u)\right\| d u \\
\quad+\left\|e^{(t-j r-r) A}\right\| \cdot\|\Gamma(r)\| \cdot\left\|\int_{j r}^{(j+1) r} A_{1} G(u) d u\right\| \\
\leq \quad \int_{j r}^{(j+1) r} C_{j}^{\prime}\|\Gamma(r)\|(u-j r)^{\gamma-1}(t-u-r)^{-\gamma} d u+M\|\Gamma(r)\| C_{j}^{\prime} \\
\leq C_{j}^{\prime}\|\Gamma(r)\| \int_{j r}^{t-r}(t-r-u)^{-\gamma}(u-j r)^{\gamma-1} d u+M\|\Gamma(r)\| C_{j}^{\prime} \\
\leq C_{j}^{\prime}\|\Gamma(r)\| B(1-\gamma, \gamma)+M\|\Gamma(r)\| C_{j}^{\prime}, \quad C_{\gamma}>0, C_{j}^{\prime}>0,
\end{aligned}
$$

where $B(\cdot, \cdot)$ is the standard Beta function. In a similar manner, we have for $t \in(n r,(n+$ 1) $r$ ] that

$$
\begin{aligned}
& \left\|\int_{(n-1) r}^{t-r} e^{(t-u-r) A} \Gamma(r) A_{1} G(u) d u\right\| \\
& \quad \leq \quad \int_{(n-1) r}^{t-r}\left\|e^{(t-u-r) A}-e^{(t-n r) A}\right\| \cdot\|\Gamma(r)\| \cdot\left\|A_{1} G(u)\right\| d u \\
& \quad+\left\|e^{(t-n r) A}\right\| \cdot\|\Gamma(r)\| \cdot\left\|\int_{(n-1) r}^{t-r} A_{1} G(u) d u\right\| \\
& \quad \leq C_{n-1}^{\prime}\|\Gamma(r)\| \int_{(n-1) r}^{t-r} \frac{(u-(n-1) r)^{\gamma}}{(t-r-u)^{\gamma}} \cdot \frac{1}{u-(n-1) r} d u+M\|\Gamma(r)\| C_{n-1}^{\prime} \\
& \quad=C_{n-1}^{\prime}\|\Gamma(r)\| B(1-\gamma, \gamma)+M\|\Gamma(r)\| C_{n-1}^{\prime}, \quad C_{n-1}^{\prime}>0 .
\end{aligned}
$$

By virtue of Eqs. 3.15, 3.16 and 3.17, we thus obtain for $t \in(n r,(n+1) r]$ that

$$
\left\|\int_{0}^{t-r} e^{(t-u-r) A} \Gamma(r) A_{1} G(u) d u\right\| \leq(M+B(1-\gamma, \gamma))\|\Gamma(r)\| \sum_{j=0}^{n-1} C_{j}^{\prime} .
$$


On the other hand, we have by using Eqs. 3.3 and 3.13 that

$$
\begin{aligned}
& \left\|\int_{t-r}^{n r} \Gamma(t-u) A_{1} G(u) d u\right\| \\
& \quad \leq \int_{t-r}^{n r}\|\Gamma(t-u)-\Gamma(r)\| \cdot\left\|A_{1} G(u)\right\| d u+\|\Gamma(r)\| \cdot\left\|\int_{t-r}^{n r} A_{1} G(u) d u\right\| \\
& \quad \leq \int_{t-r}^{n r} C_{\beta, \gamma}(u-t+r)^{\beta} \cdot \frac{C_{n-1}^{\prime}}{u-t+r} d u+\|\Gamma(r)\| C_{n-1}^{\prime} \\
& \quad \leq \frac{C_{\beta, \gamma} C_{n-1}^{\prime} r^{\beta}}{\beta}+\|\Gamma(r)\| \cdot C_{n-1}^{\prime},
\end{aligned}
$$

where $0<\beta<1-\gamma$ and $C_{\beta, \gamma}, C_{n-1}^{\prime}>0$. Combining Eqs. 3.12, 3.18 and 3.19, we thus have

$$
\left\|V_{0}(t)\right\| \leq \frac{M_{\gamma}}{t^{\gamma}}+C_{n+1}^{\prime}+C_{n+1}^{\prime \prime} \leq C_{n+1}^{\prime \prime \prime} / t^{\gamma}, \quad t \in(n r,(n+1) r],
$$

for some $C_{n+1}^{\prime \prime \prime}>0$. Now the inequality (2.8) for $t \in[0,(n+1) r]$ follows from Eqs. 3.9, 3.10, 3.20 and a similar argument to Eq. 3.7. The proof is complete now.

\section{Proof of Theorem 2.1 (b)}

We first consider $V_{0}(t)$ and $V(t), t \in[0, r]$, defined in Eq. 3.4.

Lemma 4.1 For any $0<s<t \leq r$ and $0<\beta<1-\gamma$, there exists a constant $M_{\beta, \gamma}>0$ such that

$$
\|V(t)-V(s)\| \leq M_{\beta, \gamma}(t-s)^{\beta} s^{-\beta} .
$$

Proof If condition (H1) holds, it is known by Liu [6] that Eq. 4.1 is true. Now assume that (H2) holds and we have, by definition, that for $0<s<t \leq r$,

$$
\begin{aligned}
V_{0}(t)-V_{0}(s)= & \int_{s}^{t} \Gamma(t-u) A_{1} e^{u A} d u+\int_{0}^{s}(\Gamma(t-u)-\Gamma(t)-\Gamma(s-u)+\Gamma(s)) A_{1} e^{u A} d u \\
& +(\Gamma(t)-\Gamma(s)) A_{1} A^{-1}\left(e^{s A}-I\right) \\
= & : J_{1}(s, t)+J_{2}(s, t)+J_{3}(s, t) .
\end{aligned}
$$

Note that

$$
\log (1+a) \leq a^{\alpha} / \alpha \quad \text { for any } \quad a>0,0<\alpha \leq 1 .
$$

Therefore, for $0<s<t \leq r$, it is easy to see from Eq. 4.3 that

$$
\begin{aligned}
\left\|J_{1}(s, t)\right\| & \leq \int_{s}^{t}\|\Gamma(t-u)\| \cdot\left\|A_{1} A^{-1}\right\| \cdot\left\|A e^{u A}\right\| d u \\
& \leq M\|\Gamma\|_{\infty} \cdot\left\|A_{1} A^{-1}\right\| \ln \left(1+\frac{t-s}{s}\right) \\
& \leq \frac{M\|\Gamma\|_{\infty} \cdot\left\|A_{1} A^{-1}\right\|}{\beta}(t-s)^{\beta} s^{-\beta}, \quad M>0,
\end{aligned}
$$

and in a similar manner, by Proposition 3.1, we have for $0<s<t \leq r$ that

$$
\left\|J_{3}(s, t)\right\| \leq C_{\beta, \gamma}(t-s)^{\beta} \cdot s^{-\beta}, \quad C_{\beta, \gamma}>0 .
$$


To estimate $J_{2}(\cdot \cdot)$, note that for $0<u<s<t \leq r$ and some $\delta>0$ sufficiently small, we have by using Proposition 3.1 that

$$
\begin{aligned}
\|\Gamma(t-u)-\Gamma(t)-\Gamma(s-u)+\Gamma(s)\| & \leq\|\Gamma(t-u)-\Gamma(t)\|+\|\Gamma(s-u)+\Gamma(s)\| \\
& \leq C_{\delta, \beta, \gamma}^{\prime} \cdot u^{\beta+\delta}, \quad C_{\delta, \beta, \gamma}^{\prime}>0,
\end{aligned}
$$

and

$$
\begin{aligned}
\|\Gamma(t-u)-\Gamma(t)-\Gamma(s-u)+\Gamma(s)\| & \leq\|\Gamma(t-u)-\Gamma(s-u)\|+\|\Gamma(t)-\Gamma(s)\| \\
& \leq C_{\delta, \beta, \gamma}^{\prime \prime} \cdot(t-s)^{\beta+\delta}, \quad C_{\delta, \beta, \gamma}^{\prime \prime}>0 .
\end{aligned}
$$

Hence, we have from Eqs. 4.6 and 4.7 that

$$
\begin{aligned}
\|\Gamma(t-u)-\Gamma(t)-\Gamma(s-u)+\Gamma(s)\| & =\|\Gamma(t-u)-\Gamma(t)-\Gamma(s-u)+\Gamma(s)\|^{\frac{\delta}{\beta+\delta}+\frac{\beta}{\beta+\delta}} \\
& \leq C_{\delta, \beta, \gamma}^{\prime \prime \prime}(t-s)^{\beta} \cdot u^{\delta}, \quad C_{\delta, \beta, \gamma}^{\prime \prime \prime}>0,
\end{aligned}
$$

which, in addition to Eq. 4.2, implies

$$
\begin{aligned}
\left\|J_{2}(s, t)\right\| & \leq C_{\delta, \beta, \gamma}^{\prime \prime \prime}(t-s)^{\beta} \int_{0}^{s} u^{\delta}\left\|A_{1} A^{-1}\right\| \cdot\left\|A e^{u A}\right\| d u \\
& \leq C_{\delta, \beta, \gamma}^{\prime \prime \prime}(t-s)^{\beta}\left\|A_{1} A^{-1}\right\| \int_{0}^{s} M \cdot u^{\delta-1} d u \\
& \leq M C_{\delta, \beta, \gamma}^{\prime \prime \prime} r^{\delta}\left\|A_{1} A^{-1}\right\| \delta^{-1}(t-s)^{\beta} \cdot r^{\beta} \cdot s^{-\beta} \\
& \preceq \delta, \beta, \gamma(t-s)^{\beta} \cdot s^{-\beta} .
\end{aligned}
$$

Hence, by combining Eqs. 4.4, 4.5 and 4.9, we get for $0<s<t \leq r$ that

$$
\left\|V_{0}(t)-V_{0}(s)\right\| \leq M_{\beta, \gamma}(t-s)^{\beta} s^{-\beta} \quad \text { for some } \quad M_{\beta, \gamma}>0,
$$

from which the desired result follows by the well-known Gronwall inequality and Eq. 3.4.

For $\gamma \in(0,1)$ and $0<\beta<1-\gamma$, it is known (see, Corollary 1 in [6]) that for any $0<s<t<\infty$,

$$
\left\|(-A)^{\gamma} e^{t A}-(-A)^{\gamma} e^{s A}\right\| \leq C_{\beta, \gamma}(t-s)^{\beta} \cdot s^{-\beta-\gamma} \quad \text { for some } \quad C_{\beta, \gamma}>0 .
$$

Hence, by virtue of Eqs. 3.8, 4.1 and 4.10, we obtain the estimate Eq. 2.9 in Theorem 2.1 (b) for $0<s<t \leq r$ :

$$
\begin{aligned}
\left\|(-A)^{\gamma}(G(t)-G(s))\right\| & \leq\left\|(-A)^{\gamma} e^{t A}-(-A)^{\gamma} e^{s A}\right\|+\|V(t)-V(s)\| \\
& \leq M_{\beta, \gamma}\left[(t-s)^{\beta} \cdot s^{-\beta-\gamma}+(t-s)^{\beta} s^{-\beta}\right] \\
& \leq\left(M_{\beta, \gamma}+M_{\beta, \gamma} r^{\gamma}\right)(t-s)^{\beta} \cdot s^{-\beta-\gamma},
\end{aligned}
$$

where $M_{\beta, \gamma}>0$ and $0<\beta<1-\gamma$.

Now let us show the estimates Eq. 2.10 in Theorem 2.1 (b). It suffices to show the results on each interval $[n r,(n+1) r], n \geq 1$.

(I) We first assume that condition (H1) holds. It is noticed that on the intervals $[n r,(n+$ 1) $r$, $n \geq 1$, the integral equation to be satisfied by

$$
V(t)=(-A)^{\gamma} G(t), \quad t \in[n r,(n+1) r],
$$

is

$$
V(t)=V_{0}(t)+\int_{n r}^{t} \Gamma(t-u) A_{1}(-A)^{-\gamma} V(u) d u,
$$


and $V_{0}(t)$ is given as in Eq. 3.11. We first show the Hölder continuity of $V_{0}(t)$ and $V(t)$. Let $n r<s<t \leq(n+1) r$. By virtue of Eq. 3.11, we have

$$
\begin{aligned}
V_{0}(t)-V_{0}(s)= & (-A)^{\gamma}\left(e^{t A}-e^{s A}\right) \\
& +\left(\int_{0}^{t-r} e^{(t-r-u) A} \Gamma(r) A_{1} G(u) d u-\int_{0}^{s-r} e^{(s-r-u) A} \Gamma(r) A_{1} G(u) d u\right) \\
& +\left(\int_{t-r}^{n r} \Gamma(t-u) A_{1} G(u) d u-\int_{s-r}^{n r} \Gamma(s-u) A_{1} G(u) d u\right) \\
=: & I_{1}(t, s)+I_{2}(t, s)+I_{3}(t, s) .
\end{aligned}
$$

First, by virtue of Eq. 4.10 we have

$$
\left\|I_{1}(t, s)\right\| \leq M_{\gamma}(t-s)^{\beta} s^{-\beta-\gamma} \leq \frac{M_{\gamma}}{r^{\gamma}}(t-s)^{\beta} \cdot(s-r)^{-\beta}, \quad M_{\gamma}>0 .
$$

As for the second term on the right side of Eq. 4.12, we have

$$
\begin{aligned}
I_{2}(t, s)= & \int_{s-r}^{t-r} e^{(t-r-u) A} \Gamma(r) A_{1} G(u) d u \\
& +\int_{(n-1) r}^{s-r}\left(e^{(t-r-u) A}-e^{(s-r-u) A}-e^{(t-n r) A}+e^{(s-n r) A}\right) \Gamma(r) A_{1} G(u) d u \\
& +\left(e^{(t-n r) A}-e^{(s-n r) A}\right) \int_{(n-1) r}^{s-r} \Gamma(r) A_{1} G(u) d u \\
& +\int_{0}^{(n-1) r}\left(e^{(t-r-u) A}-e^{(s-r-u) A}\right) \Gamma(r) A_{1} G(u) d u \\
=: & J_{1}(t, s)+J_{2}(t, s)+J_{3}(t, s)+J_{4}(t, s) .
\end{aligned}
$$

By virtue of Eqs. 2.2 and 2.8, we have for sufficiently small $\delta>0$ with $1-\beta-\delta>0$, $1-v-\delta>0$ that

$$
\begin{aligned}
\left\|J_{1}(t, s)\right\| & \leq\left\|(-A)^{-\delta}\right\| \int_{s-r}^{t-r}\left\|(-A)^{\delta} e^{(t-r-u) A}\right\| \cdot\|\Gamma(r)\| \cdot\left\|A_{1}(-A)^{-v}\right\| \cdot\left\|(-A)^{v} G(u)\right\| d u \\
& \leq M_{\gamma, v, \delta} \int_{s-r}^{t-r}(t-r-u)^{-\delta} \cdot \frac{C_{n, v}}{u^{\nu}} d u \\
& \preceq_{n, \gamma, \delta, v}(t-s)^{\beta} \int_{0}^{t-r}(t-r-u)^{-\beta-\delta} u^{-v} d u \\
& \preceq_{n, \gamma, \delta, v} B(1-\beta-\delta, 1-v)(t-s)^{\beta}(t-r)^{1-\beta-\delta-v} \\
& \preceq_{n, \gamma, \delta, v}(t-s)^{\beta} \cdot(s-r)^{-\beta} .
\end{aligned}
$$

To deal with $J_{2}(\cdot, \cdot)$, we have by virtue of Eq. 3.14 that for $(n-1) r<u<s-r$ and sufficiently small $\delta>0$,

$$
\begin{aligned}
\| e^{(t-r-u) A} & -e^{(s-r-u) A}-e^{(t-n r) A}+e^{(s-n r) A} \| \\
& \leq\left\|e^{(t-r-u) A}-e^{(s-r-u) A}\right\|+\left\|e^{(t-n r) A}-e^{(s-n r) A}\right\| \\
& \preceq \beta, \gamma, \delta \frac{(t-s)^{\beta+\delta}}{(s-r-u)^{\beta+\delta}}+\frac{(t-s)^{\beta+\delta}}{(s-n r)^{\beta+\delta}} \preceq_{\beta, \gamma, \delta} \frac{(t-s)^{\beta+\delta}}{(s-r-u)^{\beta+\delta}} .
\end{aligned}
$$


In a similar way, we have by Eq. 3.14 that

$$
\begin{aligned}
\| e^{(t-r-u) A} & -e^{(s-r-u) A}-e^{(t-n r) A}+e^{(s-n r) A} \| \\
& \leq\left\|e^{(t-r-u) A}-e^{(t-n r) A}\right\|+\left\|e^{(s-r-u) A}-e^{(s-n r) A}\right\| \\
& \preceq \alpha, \gamma, \delta \frac{(u-(n-1) r)^{\beta+\delta}}{(t-r-u)^{\beta+\delta}}+\frac{(u-(n-1) r)^{\beta+\delta}}{(s-r-u)^{\beta+\delta}} \\
& \preceq \beta, \gamma, \delta \frac{(u-(n-1) r)^{\beta+\delta}}{(s-r-u)^{\beta+\delta}} .
\end{aligned}
$$

Combining these two inequalities, it follows that

$$
\begin{aligned}
\| e^{(t-r-u) A} & -e^{(s-r-u) A}-e^{(t-n r) A}+e^{(s-n r) A} \| \\
& =\left\|e^{(t-r-u) A}-e^{(s-r-u) A}-e^{(t-n r) A}+e^{(s-n r) A}\right\|^{\frac{\beta}{\beta+\delta}+\frac{\delta}{\beta+\delta}} \\
& \leq C_{\beta, \delta}(t-s)^{\beta}(s-r-u)^{-(\beta+\delta)}(u-(n-1) r)^{\delta}, \quad C_{\beta, \delta}>0,
\end{aligned}
$$

for $0<\beta<1-\gamma$ and sufficiently small $\delta>0$. With the aid of Eq. 4.17, we have for sufficiently small $\delta>0$ that

$$
\begin{aligned}
&\left\|J_{2}(t, s)\right\| \leq C_{\beta, \delta} \int_{(n-1) r}^{s-r}(t-s)^{\beta}(s-r-u)^{-(\beta+\delta)}(u-(n-1) r)^{\delta} \\
& \cdot\left\|A_{1}(-A)^{-v}\right\| \cdot\|\Gamma(r)\| \cdot \frac{C_{n-1, \delta}}{u^{v}} d u \\
& \preceq \beta, \delta(t-s)^{\beta} \int_{0}^{s-r}(s-r-u)^{-(\beta+\delta)} u^{\delta-v} d u \\
& \preceq \beta, \gamma, \delta \\
& B(1-\beta-\delta, 1+\delta-v)(t-s)^{\beta}(s-r)^{-\beta} .
\end{aligned}
$$

In a similar manner, one can have by virtue of Eq. 3.14 that

$$
\begin{aligned}
\left\|J_{3}(t, s)\right\| & \leq\left\|e^{(t-n r) A}-e^{(s-n r) A}\right\| \cdot\|\Gamma(r)\| \int_{(n-1) r}^{s-r}\left\|A_{1}(-A)^{-v}\right\| \cdot\left\|(-A)^{v} G(u)\right\| d u \\
& \preceq \beta, v(t-s)^{\beta} \cdot(s-n r)^{-\beta} \int_{(n-1) r}^{s-r} u^{-v} d u \\
& \preceq \beta, v(t-s)^{\beta} \int_{0}^{s-r}(s-r-u)^{-\beta} u^{-v} d u \\
& \preceq \beta, v(t-s)^{\beta} \cdot(s-r)^{-\beta}
\end{aligned}
$$

and

$$
\begin{aligned}
\left\|J_{4}(t, s)\right\| & \leq \int_{0}^{(n-1) r}\left\|e^{(t-r-u) A}-e^{(s-r-u) A}\right\| \cdot\|\Gamma(r)\| \cdot\left\|A_{1}(-A)^{-v}\right\| \cdot\left\|(-A)^{v} G(u)\right\| d u \\
& \leq \beta, v, n(t-s)^{\beta} \int_{0}^{s-r}(s-r-u)^{-\beta} \cdot u^{-v} d u \\
& \preceq \beta, v, n B(1-\beta, 1-\gamma)(t-s)^{\beta} \cdot(s-r)^{-\beta}
\end{aligned}
$$

Combining Eqs. 4.14-4.20, we conclude that for some $M_{\beta, \gamma, \nu, n, \delta}>0$,

$$
\left\|I_{2}(t, s)\right\| \leq M_{\beta, \gamma, v, n, \delta}(t-s)^{\beta}(s-r)^{-\beta}, \quad n r<s<t \leq(n+1) r .
$$


Now we intend to consider $I_{3}(t, s)$. For sufficiently small $\delta>0$ and $0<\beta<1-\gamma$, we may obtain by virtue of Proposition 3.1 that

$$
\begin{aligned}
&\left\|I_{3}(t, s)\right\|= \| \int_{t-r}^{n r}(\Gamma(t-u)-\Gamma(s-u))\left(A_{1}(-A)^{-v}\right)(-A)^{v} G(u) d u \\
&-\int_{s-r}^{t-r} \Gamma(s-u)\left(A_{1}(-A)^{-(1-\delta)}\right)(-A)^{1-\delta} G(u) d u \| \\
& \leq \int_{t-r}^{n r}\|\Gamma(t-u)-\Gamma(s-u)\| \cdot\left\|A_{1}(-A)^{-v}\right\| \cdot\left\|(-A)^{v} G(u)\right\| d u \\
&+\int_{s-r}^{t-r}\|\Gamma(s-u)\| \cdot\left\|A_{1}(-A)^{-(1-\delta)}\right\| \cdot\left\|(-A)^{1-\delta} G(u)\right\| d u \\
& \preceq n, \beta, \gamma, v \int_{t-r}^{n r}(t-s)^{\beta} \cdot \frac{1}{u^{v}} d u+\int_{s-r}^{t-r} \frac{1}{u^{1-\delta}} d u \\
& \preceq n, \beta, \gamma, v, \delta \\
& \preceq n, \beta, \gamma, v, \delta(t-s)^{\beta} \frac{(n r)^{1-v}}{1-v}+(t-s)^{\beta}(t-r)^{-\beta+\delta} \\
& \varliminf_{n, \beta, \gamma, \delta}(t-s)^{\beta}(s-r)^{-\beta}
\end{aligned}
$$

Combining Eqs. 4.12-4.21, we thus conclude that for $n r<s<t \leq(n+1) r$,

$$
\left\|V_{0}(t)-V_{0}(s)\right\| \leq M_{n, \beta, \gamma}^{\prime}(t-s)^{\beta}(s-r)^{-\beta}, \quad M_{n, \beta, \gamma}^{\prime}>0,
$$

and further we have

$$
\|V(t)-V(s)\| \leq M_{n, \beta, \gamma}^{\prime \prime}(t-s)^{\beta}(s-r)^{-\beta}, \quad M_{n, \beta, \gamma}^{\prime \prime}>0 .
$$

(II) Now assume that condition (H2) holds. Note that on the intervals $[n r,(n+1) r]$, $n \geq 1$, the integral equation to be satisfied by

$$
V(t)=(-A)^{\gamma} G(t), \quad t \in[n r,(n+1) r],
$$

is

$$
V(t)=V_{0}(t)+\int_{n r}^{t} \Gamma(t-u) A_{1}(-A)^{-\gamma} V(u) d u,
$$

and $V_{0}(t)$, given in Eq. 3.11, can be re-written as

$$
\begin{aligned}
V_{0}(t) & -V_{0}(s) \\
=\quad & (-A)^{\gamma}\left(e^{t A}-e^{s A}\right)+\int_{s-r}^{t-r} e^{(t-r-u) A} \Gamma(r) A_{1} G(u) d u \\
& +\int_{0}^{s-r}\left(e^{(t-r-u) A}-e^{(s-r-u) A}-e^{(t-r) A}+e^{(s-r) A}\right) \Gamma(r) A_{1} G(u) d u \\
& +\left(e^{(t-r) A}-e^{(s-r) A}\right) \int_{0}^{s-r} \Gamma(r) A_{1} G(u) d u \\
& +\int_{t-r}^{n r}(\Gamma(t-u)-\Gamma(s-u)) A_{1} G(u) d u-\int_{s-r}^{t-r} \Gamma(s-u) A_{1} G(u) d u \\
=: & I_{1}(t, s)+I_{2}(t, s)+I_{3}(t, s)+I_{4}(t, s)+I_{5}(t, s)+I_{6}(t, s), \quad n r<s<t \leq(n+1) r .
\end{aligned}
$$


First, by virtue of Eq. 4.10 we have

$$
\left\|I_{1}(t, s)\right\| \leq \frac{M_{\gamma}}{r^{\gamma}}(t-s)^{\beta} \cdot(s-r)^{-\beta}, \quad M_{\gamma}>0 .
$$

It is known by Proposition 4.2 in Jeong [4] that for $i=0,1, A_{0}=A$,

$$
\left\|A_{i} G(t)\right\| \leq \frac{C_{n+1}}{t}, \quad\left\|\int_{s}^{t} A_{i} G(u) d u\right\| \leq C_{n+1}, \quad 0 \leq s<t \leq(n+1) r, \quad n \in \mathbb{N}_{0},
$$

for some $C_{n+1}>0$. Hence, for $I_{2}(t, s)$, we have by Eqs. 4.3 and 4.26 that

$$
\begin{aligned}
\left\|I_{2}(t, s)\right\| & \leq \int_{s-r}^{t-r}\left\|e^{(t-r-u) A}\right\| \cdot\|\Gamma(r)\| \cdot\left\|A_{1} A^{-1}\right\| \cdot\|A G(u)\| d u \\
& \preceq \int_{s-r}^{t-r} \frac{C_{n}}{u} d u \\
& { }_{n} \log \left(1+\frac{t-s}{s-r}\right) \\
& \preceq_{n, \beta}(t-s)^{\beta} \cdot(s-r)^{-\beta} .
\end{aligned}
$$

To handle $I_{3}(\cdot, \cdot)$, we have by virtue of Eq. 3.14 that for $0<u<s-r$ and sufficiently small $\delta>0$,

$$
\begin{aligned}
\| e^{(t-r-u) A} & -e^{(s-r-u) A}-e^{(t-r) A}+e^{(s-r) A} \| \\
& \leq\left\|e^{(t-r-u) A}-e^{(s-r-u) A}\right\|+\left\|e^{(t-r) A}-e^{(s-r) A}\right\| \\
& \preceq \beta, \gamma, \delta \frac{(t-s)^{\beta+\delta}}{(s-r-u)^{\beta+\delta}}+\frac{(t-s)^{\beta+\delta}}{(s-r)^{\beta+\delta}} \\
& \preceq \beta, \gamma, \delta \frac{(t-s)^{\beta+\delta}}{(s-r-u)^{\beta+\delta}} .
\end{aligned}
$$

In a similar way, we have by Eq. 3.14 that

$$
\begin{aligned}
\| e^{(t-r-u) A} & -e^{(s-r-u) A}-e^{(t-r) A}+e^{(s-r) A} \| \\
& \leq\left\|e^{(t-r-u) A}-e^{(t-r) A}\right\|+\left\|e^{(s-r-u) A}-e^{(s-r) A}\right\| \\
& \preceq_{\alpha, \gamma, \delta} \frac{u^{\beta+\delta}}{(t-r-u)^{\beta+\delta}}+\frac{u^{\beta+\delta}}{(s-r-u)^{\beta+\delta}} \\
& \preceq \beta, \gamma, \delta \frac{u^{\beta+\delta}}{(s-r-u)^{\beta+\delta}} .
\end{aligned}
$$

Combining Eqs. 4.28 and 4.29, we have

$$
\begin{aligned}
\| e^{(t-r-u) A} & -e^{(s-r-u) A}-e^{(t-n r) A}+e^{(s-n r) A} \| \\
& =\left\|e^{(t-r-u) A}-e^{(s-r-u) A}-e^{(t-n r) A}+e^{(s-n r) A}\right\|^{\frac{\beta}{\beta+\delta}+\frac{\delta}{\beta+\delta}} \\
& \leq C_{\beta, \delta}(t-s)^{\beta}(s-r-u)^{-(\beta+\delta)} u^{\delta}, \quad C_{\beta, \delta}>0,
\end{aligned}
$$


for $0<\beta<1-\gamma$ and sufficiently small $\delta>0$. By using Eqs. 4.26 and 4.30, we have for sufficiently small $\delta>0$ that

$$
\begin{aligned}
& \left\|I_{3}(t, s)\right\| \leq C_{\beta, \delta} \int_{0}^{s-r}(t-s)^{\beta}(s-r-u)^{-(\beta+\delta)} u^{\delta} \\
& \cdot\|\Gamma(r)\| \cdot\left\|A_{1}(-A)^{-1}\right\| \frac{C_{n-1}}{u} d u \\
& \preceq_{\beta, \delta}(t-s)^{\beta} \int_{0}^{s-r}(s-r-u)^{-(\beta+\delta)} u^{\delta-1} d u \\
& \preceq_{\beta, \delta} B(1-\beta-\delta, \delta)(t-s)^{\beta}(s-r)^{-\beta} .
\end{aligned}
$$

On the other hand, we have by using Eqs. 3.14 and 4.26 that

$$
\begin{aligned}
\left\|I_{4}(t, s)\right\| & \leq\left\|e^{(t-n r) A}-e^{(s-n r) A}\right\| \cdot\|\Gamma(r)\| \cdot\left\|A_{1} A^{-1}\right\| \cdot\left\|\int_{0}^{s-r} A G(u) d u\right\| \\
& \preceq \beta, n(t-s)^{\beta} \cdot(s-r)^{-\beta} .
\end{aligned}
$$

For $I_{5}(t, s)$, we may obtain by virtue of Proposition 3.1 and Eq. 4.26 that

$$
\begin{aligned}
\left\|I_{5}(t, s)\right\| & \leq \int_{t-r}^{n r}\|\Gamma(t-u)-\Gamma(s-u)\| \cdot\left\|A_{1} A^{-1}\right\| \cdot\|A G(u)\| d u \\
& \preceq_{\beta, \gamma}(t-s)^{\beta} \cdot \int_{t-r}^{n r} u^{-1} d u \\
& \preceq_{\beta, \gamma}(t-s)^{\beta} \ln \left(1+\frac{n r-(t-r)}{t-r}\right) \\
& \preceq_{\beta, \gamma}(t-s)^{\beta} \cdot \frac{r^{\beta}}{(t-r)^{\beta}} \\
& \preceq_{\beta, \gamma}(t-s)^{\beta} \cdot(s-r)^{-\beta} .
\end{aligned}
$$

Last, for $I_{6}(t, s)$ and $n r<s<t \leq(n+1) r$, we have by virtue of Eqs. 4.3 and 4.26 that

$$
\begin{aligned}
\left\|I_{6}(t, s)\right\| & \leq \int_{s-r}^{t-r}\|\Gamma(s-u)\| \cdot\left\|A_{1} A^{-1}\right\| \cdot\|A G(u)\| d u \\
& \preceq_{n, \beta, \delta} \int_{s-r}^{t-r} \frac{1}{u} d u \\
& \preceq_{n, \beta, \delta} \ln \left(1+\frac{t-s}{s-r}\right) \\
& \preceq_{n, \beta, \delta}(t-s)^{\beta} \cdot(s-r)^{-\beta} .
\end{aligned}
$$

Combining Eqs. 4.24-4.34, we thus conclude that

$$
\left\|V_{0}(t)-V_{0}(s)\right\| \leq M_{n, \beta, \gamma}^{\prime}(t-s)^{\beta}(s-r)^{-\beta}, \quad M_{n, \beta, \gamma}^{\prime}>0,
$$

and further

$$
\|V(t)-V(s)\| \leq M_{n, \beta, \gamma}^{\prime \prime}(t-s)^{\beta}(s-r)^{-\beta}, \quad M_{n, \beta, \gamma}^{\prime \prime}>0
$$


for $n r<s<t \leq(n+1) r$.

Finally, by combining Eqs. 4.22 and 4.35, we have for any $(n-1) r<s \leq n r<t \leq$ $(n+1) r, n>1$, that

$$
\|V(t)-V(n r)\| \preceq_{n}\left(\frac{t-n r}{n r-r}\right)^{\beta} \preceq_{n}(t-s)^{\beta}(s-r)^{-\beta},
$$

and

$$
\|V(n r)-V(s)\| \preceq_{n}\left(\frac{n r-s}{s-r}\right)^{\beta} \preceq_{n}(t-s)^{\beta}(s-r)^{-\beta} .
$$

Hence, Eq. 4.22 holds for $r<s<t \leq n r$ and $t-s<r, n>1$ with constants independent on $s$ and $t$, and further we obtain the desired estimates Eq. 2.10. The proof is thus complete.

\section{Stochastic Convolution}

Let $\{\Omega, \mathscr{F}, \mathbb{P}\}$ be a probability space equipped with some filtration $\left\{\mathscr{F}_{t}\right\}_{t \geq 0}$. Let $K$ be a separable Hilbert space and $\left\{W_{Q}(t), t \geq 0\right\}$ denote a $Q$-Wiener process with respect to $\left\{\mathscr{F}_{t}\right\}_{t \geq 0}$ in $K$, defined on $\{\Omega, \mathscr{F}, \mathbb{P}\}$ where $Q$ is a positive, self-adjoint and trace class operator on $K$. We frequently call $W_{Q}(t), t \geq 0$, a $K$-valued $Q$-Wiener process with respect to $\left\{\mathscr{F}_{t}\right\}_{t \geq 0}$ if the trace $\operatorname{Tr}(Q)<\infty$. We introduce a subspace $K_{Q}=\mathscr{R}\left(Q^{1 / 2}\right) \subset K$, the range of $Q^{1 / 2}$, which is a Hilbert space endowed with the inner product

$$
\langle u, v\rangle_{K_{Q}}=\left\langle Q^{-1 / 2} u, Q^{-1 / 2} v\right\rangle_{K} \quad \text { for any } \quad u, v \in K_{Q} .
$$

Let $\mathscr{L}_{2}\left(K_{Q}, H\right)$ be the space of all Hilbert-Schmidt operators from $K_{Q}$ into $H$. Suppose that $W(\cdot)$ is a $Q$-Wiener process in $K$ such that

$$
Q e_{j}=\lambda_{j} e_{j}, \quad j \geq 1, \quad \lambda_{j}>0,
$$

where $\left\{e_{j}\right\}$ is a complete orthonormal basis in $K$, then it is immediate that

$$
W(t)=\sum_{j=1}^{\infty} \sqrt{\lambda_{j}} w_{j}(t) e_{j}, \quad t \geq 0,
$$

where $\left\{w_{j}(\cdot)\right\}$ is a group of independent real Wiener processes.

We are concerned about the following linear stochastic retarded functional differential equation on $H$,

$$
\left\{\begin{array}{l}
d y(t)=A y(t) d t+\int_{-r}^{0} a(\theta) A_{1} y(t+\theta) d \theta d t+B d W(t), \quad t \geq 0, \\
y(0)=\phi_{0}, \quad y(\theta)=\phi_{1}(\theta), \quad \theta \in[-r, 0], \quad \phi=\left(\phi_{0}, \phi_{1}\right),
\end{array}\right.
$$

where $A, A_{1}$ are given as in Section $2, B \in \mathscr{L}_{2}\left(K_{Q}, H\right)$ and $\phi=\left(\phi_{0}, \phi_{1}\right)$ is an appropriate initial datum. It is well known that the unique mild solution $y$ of Eq. 5.2 is represented as

$$
y(t)=G(t) \phi_{0}+\int_{-r}^{0} U_{t}(\theta) \phi_{1}(\theta) d \theta+\int_{0}^{t} G(t-s) B d W(s), \quad t \geq 0,
$$


where

$$
U_{t}(\theta)=\int_{-r}^{\theta} G(t-\theta+\tau) a(\tau) A_{1} d \tau, \quad \theta \in[-r, 0],
$$

with the initial condition $y(0)=\phi_{0}$ and $y(\theta)=\phi_{1}(\theta), \theta \in[-r, 0)$. In particular, if $\phi=(0,0)$, then the unique mild solution Eq. 5.3 is the so-called stochastic convolution process

$$
y(t):=W_{G}(t)=\int_{0}^{t} G(t-s) B d W(s), \quad t \geq 0 .
$$

For simplicity, let us assume at present that $K=H$ and $B=I$ in Eq. 5.2.

Theorem 5.1 Let $T>0$ and $\operatorname{Tr}(Q)<\infty$. Suppose that condition (H1) (or (H2)) holds, then for $v \leq \gamma<1 / 2($ or $0<\gamma<1 / 2)$ and $0<\beta<\frac{1}{2}-\gamma$, the trajectories of $W_{G}$ are in $C^{\beta}\left([0, T], \mathscr{D}\left((-A)^{\gamma}\right)\right)$, the space of all $\mathscr{D}\left((-A)^{\gamma}\right)$-valued, Hölder continuous functions with order $\beta$.

Proof We intend to use a Kolmogorov test type of argument (see, e.g., Theorem 3.5 in Da Prato and Zabczyk [2]). For any $0 \leq s<t \leq T$, by definition, it is easy to see that

$$
\begin{aligned}
& \mathbb{E}\left\|(-A)^{\gamma} W_{G}(t)-(-A)^{\gamma} W_{G}(s)\right\|_{H}^{2} \\
= & \sum_{k=1}^{\infty} \lambda_{k} \int_{s}^{t}\left\|(-A)^{\gamma} G(t-u) e_{k}\right\|_{H}^{2} d u+\sum_{k=1}^{\infty} \lambda_{k} \int_{0}^{s}\left\|(-A)^{\gamma}[G(t-u)-G(s-u)] e_{k}\right\|_{H}^{2} d u \\
= & I_{1}+I_{2} .
\end{aligned}
$$

Now we estimate $I_{1}$ and $I_{2}$, respectively. Since $0<\beta<\frac{1}{2}-\gamma$, it follows that $1-2 \gamma>$ $2 \beta>0$. By using Theorem 2.1 (a), we thus have

$$
\begin{aligned}
I_{1} & \leq \operatorname{Tr}(Q) \int_{0}^{t-s}\left\|(-A)^{\gamma} G(v)\right\|^{2} d v \\
& \leq \operatorname{Tr}(Q) \int_{0}^{t-s} \frac{C_{0, \gamma}^{2}}{v^{2 \gamma}} d v=\frac{\operatorname{Tr}(Q) C_{0, \gamma}^{2}}{1-2 \gamma}(t-s)^{1-2 \gamma}, \quad C_{0, \gamma}>0 .
\end{aligned}
$$

Note that $1-2 \beta-2 \gamma>0$. If $s \leq r$, we employ Theorem 2.1 (b) to obtain

$$
\begin{aligned}
I_{2} & =\sum_{k=1}^{\infty} \lambda_{k} \int_{0}^{s}\left\|(-A)^{\gamma}[G(t-s+v)-G(v)] e_{k}\right\|_{H}^{2} d v \\
& \leq \operatorname{Tr}(Q) \int_{0}^{r} \frac{C_{0, \beta, \gamma}^{2}(t-s)^{2 \beta}}{v^{2 \beta+2 \gamma}} d v \\
& \leq \operatorname{Tr}(Q) \frac{C_{0, \beta, \gamma}^{2} r^{1-2 \beta-2 \gamma}}{1-2 \beta-2 \gamma}(t-s)^{2 \beta}, \quad C_{0, \beta, \gamma}>0 .
\end{aligned}
$$


If $s>r$, we similarly employ Theorem 2.1 (b) to obtain

$$
\begin{aligned}
I_{2}= & \sum_{k=1}^{\infty} \lambda_{k} \int_{0}^{s}\left\|(-A)^{\gamma}[G(t-s+v)-G(v)] e_{k}\right\|_{H}^{2} d v \\
= & \sum_{k=1}^{\infty} \lambda_{k} \int_{0}^{r}\left\|(-A)^{\gamma}[G(t-s+v)-G(v)] e_{k}\right\|_{H}^{2} d v \\
& +\sum_{k=1}^{\infty} \lambda_{k} \int_{r}^{s}\left\|(-A)^{\gamma}[G(t-s+v)-G(v)] e_{k}\right\|_{H}^{2} d v \\
\leq & \operatorname{Tr}(Q)\left(\int_{0}^{r} \frac{C_{0, \beta, \gamma}^{2}(t-s)^{2 \beta}}{v^{2 \beta+2 \gamma}} d v+\int_{r}^{s} \frac{C_{1, \beta, \gamma}^{2}(t-s)^{2 \beta}}{(v-r)^{2 \beta}} d v\right) \\
\leq & \operatorname{Tr}(Q)\left(\frac{C_{0, \beta, \gamma}^{2} r^{1-2 \beta-2 \gamma}}{1-2 \beta-2 \gamma}+\frac{C_{1, \beta, \gamma}^{2} r^{1-2 \beta}}{1-2 \beta}\right)(t-s)^{2 \beta}, \quad C_{0, \beta, \gamma}>0, C_{1, \beta, \gamma}>0 .
\end{aligned}
$$

Hence, by substituting Eqs. 5.6, 5.7 and 5.8 into 5.5, we obtain

$$
\begin{aligned}
\mathbb{E}\left\|(-A)^{\gamma}\left(W_{G}(t)-W_{G}(s)\right)\right\|_{X}^{2} & \preceq T, \beta, \gamma(t-s)^{1-2 \gamma}+(t-s)^{2 \beta} \\
& \preceq T, \beta, \gamma(t-s)^{2 \beta} .
\end{aligned}
$$

Since $(-A)^{\gamma} W_{G}(t)-(-A)^{\gamma} W_{G}(s)$ is Gaussian, for any integer $m \geq 1$, it follows by Eq. 5.9 that

$$
\mathbb{E}\left\|(-A)^{\gamma}\left(W_{G}(t)-W_{G}(s)\right)\right\|^{2 m} \preceq T, \beta, \gamma, m(t-s)^{2 m \beta}, \quad \forall 0 \leq s<t \leq T .
$$

So, by the well-known Kolmogorov test, $(-A)^{\gamma} W_{G}(\cdot)$ is $\alpha_{m}$-Hölder continuous with order

$$
\alpha_{m}=\frac{2 m \beta-1}{2 m} \text {. }
$$

Since $m$ is arbitrary, the trajectories of process $(-A)^{\gamma} W_{G}$ are thus in $C^{\beta}([0, T] ; H)$.

\section{Delays in the Highest-Order Derivatives}

In the remainder of this work, we assume that the operators $A$ and $A_{1}$ in Eq. 5.2 are diagonal with respect to the complete orthonormal basis $\left\{e_{k}\right\}$ given in Eq. 5.1 such that

$$
A e_{k}=-\mu_{k} e_{k}, \quad A_{1} e_{k}=\tilde{\mu}_{k} e_{k},
$$

for some numbers $\mu_{k}>0, \tilde{\mu}_{k} \in \mathbb{R}, k \in \mathbb{N}=\{1,2, \cdots\}$. For $\alpha, \beta \in \mathbb{R}$, let $g(t), t \geq 0$, denote the unique solution of the following differential equation with delay

$$
\left\{\begin{array}{l}
g^{\prime}(t)+\alpha g(t)=\beta \int_{-r}^{0} a(\theta) g(t+\theta) d \theta, \quad t \geq 0, \\
g(0)=1, \quad g(\theta)=0, \quad \theta \in[-r, 0),
\end{array}\right.
$$

and the fundamental solution $G$ is determined by

$$
G(t) e_{k}=g\left(\mu_{k}, t\right) e_{k}, \quad k \in \mathbb{N},
$$


where $g\left(\mu_{k}, \cdot\right)$ is the unique solution to Eq. 6.2 with $\alpha=\mu_{k}$ and $\beta=\tilde{\mu}_{k}, k \in \mathbb{N}$. Moreover, the stochastic convolution $W_{G}(\cdot)$ is given at present by

$$
W_{G}(t)=\sum_{k=1}^{\infty} \sqrt{\lambda_{k}} \int_{0}^{t} g\left(\mu_{k}, t-s\right) e_{k} d w_{k}(s), \quad t \geq 0 .
$$

Proposition 6.1 Assume that function a(.) is Hölder continuous. For any constant $\gamma \in$ $(0,1)$, it holds true that for each $k \in \mathbb{N}$,

$$
\int_{s}^{t} g^{2}\left(\mu_{k}, u\right) d u \leq M_{n, \gamma} \cdot \mu_{k}^{-\gamma}(t-s)^{1-\gamma} \quad \text { for all } \quad 0 \leq s<t \leq n r
$$

where $M_{n, \gamma}>0$ are some numbers depending on $n$ and $\gamma$.

Proof By virtue of Eqs. 6.1 and 6.3, it is immediate that for each $k \in \mathbb{N}$ and $t \geq 0$,

$$
(-A)^{\gamma / 2} G(t) e_{k}=g\left(\mu_{k}, t\right)(-A)^{\gamma / 2} e_{k}=\mu_{k}^{\gamma / 2} g\left(\mu_{k}, t\right) e_{k} .
$$

This implies by virtue of Eq. 2.8 in Theorem 2.1 (a) that for each $k \in \mathbb{N}$,

$$
\begin{aligned}
\mu_{k}^{\gamma} g^{2}\left(\mu_{k}, t\right) & =\left\|\mu_{k}^{\gamma / 2} g\left(\mu_{k}, t\right) e_{k}\right\|_{H}^{2} \\
& =\left\|(-A)^{\gamma / 2} G(t) e_{k}\right\|_{H}^{2} \\
& \leq\left\|(-A)^{\gamma / 2} G(t)\right\|^{2} \cdot\left\|e_{k}\right\|_{H}^{2} \\
& \leq \frac{C_{n, \gamma}^{2}}{t^{\gamma}}, \quad \forall t \in(0, n r],
\end{aligned}
$$

for some $C_{n, \gamma}>0$, which immediately implies for each $k \in \mathbb{N}$ that

$$
g^{2}\left(\mu_{k}, t\right) \leq C_{n, \gamma}^{2} \mu_{k}^{-\gamma} t^{-\gamma}, \quad \forall t \in(0, n r]
$$

Hence, we further have for any $0 \leq s<t \leq n r, n \in\{1,2, \cdots\}$, that

$$
\begin{aligned}
\int_{s}^{t} g^{2}\left(\mu_{k}, u\right) d u & \leq C_{n, \gamma}^{2} \cdot \mu_{k}^{-\gamma} \int_{s}^{t} u^{-\gamma} d u \\
& \leq \frac{C_{n, \gamma}^{2} \cdot \mu_{k}^{-\gamma}}{1-\gamma}\left(t^{1-\gamma}-s^{1-\gamma}\right) .
\end{aligned}
$$

Note that for any real numbers $a \geq b \geq 0$ and $0<\delta \leq 1$, we have

$$
a^{\delta}-b^{\delta} \leq(a-b)^{\delta} \text {. }
$$

It thus follows from Eqs. 6.7 and 6.8 that for any $0 \leq s<t \leq n r, n \in\{1,2, \cdots\}$,

$$
\int_{s}^{t} g^{2}\left(\mu_{k}, u\right) d u \leq \frac{C_{n, \gamma}^{2} \cdot \mu_{k}^{-\gamma}}{1-\gamma}(t-s)^{1-\gamma}
$$

as desired. The proof is thus complete.

Proposition 6.2 Assume that function $a(\cdot)$ is Hölder continuous. Let $\gamma \in(0,1)$ and $0<$ $\beta<1-\gamma$, then for any $0 \leq s<t \leq n r, n \in\{1,2, \cdots\}$, there exists a number $M_{\gamma, \beta, n}>0$ such that

$$
\int_{0}^{s}\left[g\left(\mu_{k}, t-u\right)-g\left(\mu_{k}, s-u\right)\right]^{2} d u \leq M_{\gamma, \beta, n} \cdot \mu_{k}^{-\gamma}(t-s)^{\beta}, \quad k \in \mathbb{N} .
$$


Proof First note that for any $k \in \mathbb{N}, 0 \leq s<t \leq n r$ and $v>0$,

$$
\begin{aligned}
(-A)^{\gamma / 2}(G(t-s+v)-G(v)) e_{k} & =\left(g\left(\mu_{k}, t-s+v\right)-g\left(\mu_{k}, v\right)\right)(-A)^{\gamma / 2} e_{k} \\
& =\mu_{k}^{\gamma / 2}\left(g\left(\mu_{k}, t-s+v\right)-g\left(\mu_{k}, v\right)\right) e_{k},
\end{aligned}
$$

where $\left\{e_{k}\right\}$ is the orthonormal basis of $H$ in Eq. 6.1. Hence, by virtue of Eq. 2.9 in Theorem 2.1 (b) we have for any $0 \leq s<t \leq n r$ and $0<v \leq r$ that

$$
\begin{aligned}
\mu_{k}^{\gamma}\left[g\left(\mu_{k}, t-s+v\right)-g\left(\mu_{k}, v\right)\right]^{2} & =\left\|\mu_{k}^{\gamma / 2}\left[g\left(\mu_{k}, t-s+v\right)-g\left(\mu_{k}, v\right)\right] e_{k}\right\|_{H}^{2} \\
& =\left\|(-A)^{\gamma / 2}(G(t-s+v)-G(v)) e_{k}\right\|_{H}^{2} \\
& \leq\left\|(-A)^{\gamma / 2}(G(t-s+v)-G(v))\right\|^{2} \cdot\left\|e_{k}\right\|_{H}^{2} \\
& \leq C_{n, \beta, \gamma}^{2} \frac{(t-s)^{\beta}}{v^{\beta+\gamma}}, \quad C_{n, \beta, \gamma}>0
\end{aligned}
$$

or similarly, for any $0 \leq s<t \leq n r$ and $v>r$ that

$$
\mu_{k}^{\gamma}\left[g\left(\mu_{k}, t-s+v\right)-g\left(\mu_{k}, v\right)\right]^{2} \leq C_{n, \beta, \gamma}^{2} \frac{(t-s)^{\beta}}{(v-r)^{\beta}}, \quad C_{n, \beta, \gamma}>0 .
$$

If $s \in[0, r]$, it is easy to see from Eq. 6.10 that

$$
\begin{aligned}
\int_{0}^{s}\left[g\left(\mu_{k}, t-u\right)-g\left(\mu_{k}, s-u\right)\right]^{2} d u & =\int_{0}^{s}\left[g\left(\mu_{k}, t-s+v\right)-g\left(\mu_{k}, v\right)\right]^{2} d v \\
& \leq \mu_{k}^{-\gamma}(t-s)^{\beta} C_{n, \beta, \gamma}^{2} \int_{0}^{s} v^{-\beta-\gamma} d v \\
& =\frac{\mu_{k}^{-\gamma}}{1-\beta-\gamma} C_{n, \beta, \gamma}^{2} \cdot r^{1-\beta-\gamma}(t-s)^{\beta} \\
& =: C_{\gamma, \beta, n} \cdot \mu_{k}^{-\gamma}(t-s)^{\beta}, \quad 0 \leq s<t \leq n r .
\end{aligned}
$$

On the other hand, if $s>r$, one can similarly have from Eqs. 6.10 and 6.11 that

$$
\begin{aligned}
& \int_{0}^{s}\left[g\left(\mu_{k}, t-u\right)-g\left(\mu_{k}, s-u\right)\right]^{2} d u \\
= & \int_{0}^{s}\left[g\left(\mu_{k}, t-s+v\right)-g\left(\mu_{k}, v\right)\right]^{2} d v \\
\leq & \int_{r}^{s}\left[g\left(\mu_{k}, t-s+v\right)-g\left(\mu_{k}, v\right)\right]^{2} d v+\int_{0}^{r}\left[g\left(\mu_{k}, t-s+v\right)-g\left(\mu_{k}, v\right)\right]^{2} d v \\
\leq & \mu_{k}^{-\gamma}(t-s)^{\beta} C_{n, \beta, \gamma}^{2} \int_{r}^{s}(v-r)^{-\beta} d v+\mu_{k}^{-\gamma}(t-s)^{\beta} C_{0, \beta, \gamma}^{2} \int_{0}^{r} v^{-\beta-\gamma} d v \\
= & \frac{\mu_{k}^{-\gamma}}{1-\beta} C_{n, \beta, \gamma}^{2} \cdot[(n-1) r]^{1-\beta}(t-s)^{\beta}+\frac{\mu_{k}^{-\gamma}}{1-\beta-\gamma} C_{0, \beta, \gamma}^{2} \cdot r^{1-\beta-\gamma}(t-s)^{\beta} \\
= & : M_{\gamma, \beta, n} \cdot \mu_{k}^{-\gamma}(t-s)^{\beta}, \quad 0 \leq s<t \leq n r,
\end{aligned}
$$

where

$$
M_{\gamma, \beta, n}=\frac{1}{1-\beta} C_{n, \beta, \gamma}^{2} \cdot[(n-1) r]^{1-\beta}+\frac{1}{1-\beta-\gamma} C_{0, \beta, \gamma}^{2} \cdot r^{1-\beta-\gamma}>0 .
$$

The proof is thus complete. 
Now we are in a position to establish our regularity properties for the stochastic convolution (5.4) with $B=I$. Recall that $Q e_{k}=\lambda_{k} e_{k}, \lambda_{k}>0$, and $A e_{k}=-\mu_{k} e_{k}, \mu_{k}>0$, $k \geq 1$.

Theorem 6.1 Suppose that $\gamma \in(0,1)$ and

$$
\sum_{k=1}^{\infty} \frac{\lambda_{k}}{\mu_{k}^{\gamma}}<\infty
$$

Then the trajectories of $H$-valued process $W_{G}(t), t \geq 0$, in Eq. 5.4 are almost surely $\alpha$ Hölder continuous with respect to $t$ for

$$
0<\alpha<\frac{1-\gamma}{2}
$$

Proof It suffices to show the theorem for any $0 \leq s<t \leq n r, n \in \mathbb{N}$. To this end, we first have

$$
W_{G}(t)-W_{G}(s)=\int_{s}^{t} G(t-u) d W(u)+\int_{0}^{s}[G(t-u)-G(s-u)] d W(u) .
$$

Since the integrals are independent, it follows that

$$
\begin{aligned}
\mathbb{E}\left(\left\|W_{G}(t)-W_{G}(s)\right\|_{H}^{2}\right) & \leq \sum_{k=1}^{\infty} \lambda_{k} \int_{s}^{t} g^{2}\left(\mu_{k}, u\right) d u+\sum_{k=1}^{\infty} \lambda_{k} \int_{0}^{s}\left(g\left(\mu_{k}, t-u\right)-g\left(\mu_{k}, s-u\right)\right)^{2} d u \\
& =: J_{1}(t, s)+J_{2}(t, s) .
\end{aligned}
$$

By virtue of Propositions 6.1 and 6.2, we have for $0 \leq s<t \leq n r$ that

$$
J_{1}(t, s) \leq \sum_{k=1}^{\infty} \lambda_{k} \cdot C_{n, \gamma} \cdot \mu_{k}^{-\gamma}(t-s)^{1-\gamma} \leq\left(M_{\gamma, \beta, n} r^{1-\gamma-\beta} \sum_{k=1}^{\infty} \frac{\lambda_{k}}{\mu_{k}^{\gamma}}\right)(t-s)^{\beta}, \quad M_{\gamma, \beta, n}>0,
$$

and

$$
J_{2}(t, s) \leq\left(M_{\gamma, \beta, n} \sum_{k=1}^{\infty} \frac{\lambda_{k}}{\mu_{k}^{\gamma}}\right)(t-s)^{\beta}, \quad M_{\gamma, \beta, n}>0,
$$

where $0<\beta<1-\gamma$. Since $W_{G}(t)-W_{G}(s)$ is Gaussian, then for any $m \in \mathbb{N}$, there exists a constant $C_{m}>0$ such that

$$
\mathbb{E}\left(\left\|W_{G}(t)-W_{G}(s)\right\|_{H}^{2 m}\right) \leq C_{m}\left[\left(C_{n, \gamma} r^{1-\gamma-\beta}+C_{\gamma, \beta, n}\right) \sum_{k=1}^{\infty} \frac{\lambda_{k}}{\mu_{k}^{\gamma}}\right]^{m}(t-s)^{m \beta} .
$$

Choosing $m$ such that $m \beta>1$ and applying the well-known Kolmogorov test, one can find that $W_{G}(t)$ is $\alpha$-Hölder continuous in $H$ for

$$
\alpha=\frac{\beta}{2}-\frac{1}{2 m} \text {. }
$$

Since $m$ is arbitrary, one can obtain the desired result by letting $\beta \uparrow 1-\gamma$.

\section{Regularity in Space of Continuous Functions}

In this section, we consider the continuity of the stochastic convolution $W_{G}(t), t \geq 0$, in a smaller Banach space of $H$. 
To this end, we assume that $H=L^{2}(\mathcal{O})$ where $\mathcal{O}$ is a bounded open subset of $\mathbb{R}^{d}$ with smooth boundary $\partial \mathcal{O}$. Suppose that we are given a self-adjoint negative operator $A$ in $L^{2}(\mathcal{O})$ such that

$$
A e_{k}=-\mu_{k} e_{k}, \quad k \in \mathbb{N},
$$

where $\left\{e_{k}\right\}_{k=1}^{\infty} \subset \mathscr{D}(A)$ is the complete orthonormal basis of $H$ and the sequence of positive numbers $\left\{\mu_{k}\right\}_{k=1}^{\infty}$ satisfies

$$
\mu_{k} \geq \mu_{0}>0 \quad \text { for some } \quad \mu_{0}>0,
$$

and

$$
\sum_{k=1}^{\infty} \frac{\lambda_{k}}{\mu_{k}}<\infty
$$

We set $W_{G}(t)(\xi)=W_{G}(t, \xi)$ and write the stochastic convolution as

$$
W_{G}(t, \xi)=\sum_{k=1}^{\infty} \sqrt{\lambda_{k}} \int_{0}^{t} g\left(\mu_{k}, t-s\right) e_{k}(\xi) d w_{k}(s), \quad t \geq 0, \quad \xi \in \mathcal{O} .
$$

For the basis $\left\{e_{k}\right\}_{k=1}^{\infty}$, we suppose that there exists a constant $M>0$ such that

$$
\begin{aligned}
& \left\{e_{k}\right\} \subset C(\overline{\mathcal{O}}), \quad\left|e_{k}(\xi)\right| \leq M, \quad k \in \mathbb{N}, \quad \xi \in \mathcal{O}, \\
& \left|\nabla e_{k}(\xi)\right| \leq M \mu_{k}^{1 / 2}, \quad k \in \mathbb{N}, \quad \xi \in \mathcal{O} .
\end{aligned}
$$

Further, it is known (see (5.46) in Da Prato and Zabczyk [2]) that for each $\kappa \in[0,1]$, there exists a constant $C_{\kappa}>0$ such that

$$
\left|e_{k}(\xi)-e_{k}(\eta)\right|^{2} \leq C_{\kappa} \mu_{k}^{\kappa}|\xi-\eta|^{2 \kappa}, \quad \xi, \eta \in \mathcal{O}, \quad k \in \mathbb{N} .
$$

Theorem 7.1 If there exists a number $\delta \in(0,1)$ such that

$$
\sum_{k=1}^{\infty} \frac{\lambda_{k}}{\mu_{k}^{1-\delta}}<\infty
$$

then the process $W_{G}(t)$ has a version $W_{G}(t, \xi), t \geq 0, \xi \in \overline{\mathcal{O}}$, whose trajectories are almost surely $\alpha$-Hölder continuous with respect to $(t, \xi)$ for

$$
0<\alpha<\frac{\delta}{2} \text {. }
$$

In particular, the process $W_{G}(t)$ has a $C(\overline{\mathcal{O}})$-valued version with $\alpha$-Hölder continuous paths for $0<\alpha<\delta / 2$.

Proof It suffices to prove this theorem for any $0 \leq s<t \leq N r$ and fixed $N \in \mathbb{N}$. First, by virtue of Eqs. 6.5 and 7.3 we have that for $\varepsilon>0$ small enough with $\delta-\varepsilon>0$,

$$
\begin{aligned}
\sum_{k=1}^{\infty} \lambda_{k} \int_{0}^{t} \mu_{k}^{\delta-\varepsilon} g^{2}\left(\mu_{k}, s\right) d s & \leq M_{\delta, \varepsilon} \sum_{k=1}^{\infty} \lambda_{k} \mu_{k}^{\delta-\varepsilon-\left(1-\frac{\varepsilon}{2}\right)} \cdot(N r)^{\varepsilon / 2} \\
& \leq \frac{M_{\delta, \varepsilon}(N r)^{\varepsilon / 2}}{\mu_{0}^{\varepsilon / 2}} \sum_{k=1}^{\infty} \frac{\lambda_{k}}{\mu_{k}^{1-\delta}}<\infty
\end{aligned}
$$


Since $0<\delta<1$, it follows from Eqs. 7.2, 7.3 and 7.4 that for any $0 \leq t \leq N r$,

$$
\begin{aligned}
\mathbb{E}\left|W_{G}(t, \xi)-W_{G}(t, \eta)\right|^{2} & =\sum_{k=1}^{\infty} \lambda_{k} \int_{0}^{t} g^{2}\left(\mu_{k}, t-s\right)\left|e_{k}(\xi)-e_{k}(\eta)\right|^{2} d s \\
& \leq \sum_{k=1}^{\infty} \lambda_{k} \int_{0}^{t} g^{2}\left(\mu_{k}, t-s\right) C_{\delta, \varepsilon} \mu_{k}^{\delta-\varepsilon} \cdot|\xi-\eta|^{2(\delta-\varepsilon)} d s \\
& \leq C_{\delta, \varepsilon} M_{\delta, \varepsilon}\left\{\left(\frac{N r}{\mu_{0}}\right)^{\varepsilon / 2} \sum_{k=1}^{\infty} \frac{\lambda_{k}}{\mu_{k}^{1-\delta}}\right\}|\xi-\eta|^{2(\delta-\varepsilon)}, \quad C_{\delta, \varepsilon}>0 .
\end{aligned}
$$

On the other hand, for any $0 \leq s<t \leq N r$, we have

$$
\begin{aligned}
& \mathbb{E}\left|W_{G}(t, \xi)-W_{G}(s, \xi)\right|^{2} \\
\leq & \sum_{k=1}^{\infty} \lambda_{k} \int_{s}^{t} g^{2}\left(\mu_{k}, t-u\right)\left|e_{k}(\xi)\right|^{2} d u+\sum_{k=1}^{\infty} \lambda_{k} \int_{0}^{s}\left|\left[g\left(\mu_{k}, t-u\right)-g\left(\mu_{k}, s-u\right)\right] e_{k}(\xi)\right|^{2} d u \\
= & : I_{1}(t, s, \xi)+I_{2}(t, s, \xi) .
\end{aligned}
$$

To estimate $I_{1}(t, s, \xi)$, we have, by analogy with Eq. 6.6, that for each $k \in \mathbb{N}$ and the above $\varepsilon>0$ small enough,

$$
g^{2}\left(\mu_{k}, t\right) \leq M_{\delta, \varepsilon}^{2} \mu_{k}^{\delta-\varepsilon-1} t^{\delta-\varepsilon-1}, \quad \forall t \in[0, N r], \quad M_{\delta, \varepsilon}>0 .
$$

Hence, it follows that for $0 \leq s<t \leq N r$,

$$
\begin{aligned}
\int_{s}^{t} g^{2}\left(\mu_{k}, u\right) d u & \leq M_{\delta, \varepsilon}^{2} \cdot \mu_{k}^{\delta-\varepsilon-1} \int_{s}^{t} u^{\delta-\varepsilon-1} d u \\
& \leq \frac{M_{\delta, \varepsilon}^{2}}{\delta-\varepsilon} \frac{1}{\mu_{0}^{\varepsilon}} \cdot \frac{1}{\mu_{k}^{1-\delta}}(t-s)^{\delta-\varepsilon}
\end{aligned}
$$

Finally, we have for $0 \leq s<t \leq N r$ that

$$
\begin{aligned}
I_{1}(t, s, \xi) & =\sum_{k=1}^{\infty} \lambda_{k} \int_{0}^{t-s} g^{2}\left(\mu_{k}, v\right) d v \\
& \leq \frac{M_{\delta, \varepsilon}}{(\delta-\varepsilon) \mu_{0}^{\varepsilon}} \sum_{k=1}^{\infty} \frac{\lambda_{k}}{\mu_{k}^{1-\delta}} \cdot(t-s)^{\delta-\varepsilon} .
\end{aligned}
$$

To estimate $I_{2}(t, s, \xi)$, since $0<\delta<1$, by analogy with Eq. 6.10, one can get that for each $k \in \mathbb{N}, 0 \leq s<t \leq N r$ and $v>0$ with $\varepsilon>0$ small enough,

$$
\mu_{k}^{1-\delta+\frac{\varepsilon}{2}}\left[g\left(\mu_{k}, t-s+v\right)-g\left(\mu_{k}, v\right)\right]^{2} \leq C_{\delta, \varepsilon}^{2} \frac{(t-s)^{\delta-\varepsilon}}{v^{1-\delta+\frac{\varepsilon}{2}+\delta-\varepsilon}} \quad \text { if } \quad 0<v \leq r,
$$

and

$$
\mu_{k}^{1-\delta+\frac{\varepsilon}{2}}\left[g\left(\mu_{k}, t-s+v\right)-g\left(\mu_{k}, v\right)\right]^{2} \leq C_{\delta, \varepsilon}^{2} \frac{(t-s)^{\delta-\varepsilon}}{(v-r)^{\delta-\varepsilon}} \quad \text { if } \quad v>r,
$$


for some $C_{\delta, \varepsilon}>0$. If $s \in[0, r]$, then we have by Eq. 7.9 that

$$
\begin{aligned}
I_{2}(t, s, \xi) & \leq \sum_{k=1}^{\infty} \lambda_{k} \int_{0}^{s}\left[g\left(\mu_{k}, t-s+v\right)-g\left(\mu_{k}, v\right)\right]^{2} d v \\
& \leq \frac{C_{\delta, \varepsilon}^{2} n(N r)^{\varepsilon / 2}}{\varepsilon \mu_{0}^{\varepsilon / 2}} \sum_{k=1}^{\infty} \frac{\lambda_{k}}{\mu_{k}^{1-\delta}} \cdot(t-s)^{\delta-\varepsilon} .
\end{aligned}
$$

If $s>r$, then we have by Eqs. 7.9 and 7.10 that

$$
\begin{aligned}
I_{2}(t, s, \xi) \leq & \sum_{k=1}^{\infty} \lambda_{k} \int_{0}^{s}\left[g\left(\mu_{k}, t-s+v\right)-g\left(\mu_{k}, v\right)\right]^{2} d v \\
\leq & \sum_{k=1}^{\infty} \lambda_{k} \int_{r}^{s}\left[g\left(\mu_{k}, t-s+v\right)-g\left(\mu_{k}, v\right)\right]^{2} d v \\
& +\sum_{k=1}^{\infty} \lambda_{k} \int_{0}^{r}\left[g\left(\mu_{k}, t-s+v\right)-g\left(\mu_{k}, v\right)\right]^{2} d v \\
\leq & C_{\delta, \varepsilon}^{\prime} \frac{(N r)^{\varepsilon / 2}+(N r)^{1-\delta+\varepsilon}}{\varepsilon \mu_{0}^{\varepsilon / 2}} \sum_{k=1}^{\infty} \frac{\lambda_{k}}{\mu_{k}^{1-\delta}} \cdot(t-s)^{\delta-\varepsilon}, \quad C_{\delta, \varepsilon}^{\prime}>0 .
\end{aligned}
$$

Combining Eqs. 7.8 and 7.12, we have the conclusion that there exists $C_{\delta, \varepsilon}^{\prime \prime}>0$ such that

$$
\mathbb{E}\left|W_{G}(t, \xi)-W_{G}(s, \xi)\right|^{2} \leq C_{\delta, \varepsilon}^{\prime \prime}(t-s)^{\delta-\varepsilon}, \quad \text { for any } \quad 0 \leq s<t \leq N r
$$

Further, for any $0 \leq s<t \leq N r$, there exists some $C_{\delta, \varepsilon}^{\prime \prime \prime}>0$ such that

$$
\mathbb{E}\left|W_{G}(t, \xi)-W_{G}(s, \eta)\right|^{2} \leq C_{\delta, \varepsilon}^{\prime \prime \prime}\left[|\xi-\eta|^{2}+(t-s)\right]^{\delta-\varepsilon} .
$$

Since $W_{G}(t, \xi)-W_{G}(s, \eta)$ is a Gaussian random variable, for any $m \in \mathbb{N}$ we thus have for some number $c_{m, \delta, \varepsilon}>0$ that

$$
\mathbb{E}\left|W_{G}(t, \xi)-W_{G}(s, \eta)\right|^{2 m} \leq c_{m, \delta, \varepsilon}\left[|\xi-\eta|^{2}+(t-s)\right]^{m(\delta-\varepsilon)} .
$$

Choosing $m$ such that $m(\delta-\varepsilon)>1$ and applying the well-known Kolmogorov test to random fields, we can find that $W_{G}(\cdot, \cdot)$ is $\alpha$-Hölder continuous for

$$
\alpha=\frac{\delta-\varepsilon}{2}-\frac{1}{2 m} \text {. }
$$

Let $m \rightarrow \infty$ and $\varepsilon \rightarrow 0$, then we have the desired result.

Example 7.1 Assume that $\mathcal{O}=[0, \pi]^{d} \subset \mathbb{R}^{d}, d \in \mathbb{N}$. Let $A$ be the linear operator

$$
\left\{\begin{array}{l}
\mathscr{D}(A)=H^{2}(\mathcal{O}) \cap H_{0}^{1}(\mathcal{O}), \\
A u=\Delta u, \quad \forall u \in \mathscr{D}(A),
\end{array}\right.
$$


where $\Delta$ represents the Laplace operator, then

$$
\begin{aligned}
e_{n_{1}, \cdots, n_{d}}(\xi) & =\left(\frac{2}{\pi}\right)^{d / 2} \sin \left(n_{1} \xi_{1}\right) \cdots \sin \left(n_{d} \xi_{d}\right), \\
\left|\nabla e_{n_{1}, \cdots, n_{d}}(\xi)\right| & \leq\left(\frac{2}{\pi}\right)^{d / 2}\left(\left|n_{1}\right|+\cdots+\left|n_{d}\right|\right) \\
& \leq\left(\frac{2}{\pi}\right)^{d / 2} d^{1 / 2} \sqrt{n_{1}^{2}+\cdots+n_{d}^{2}} \\
\mu_{n_{1}, \cdots, n_{d}} & =n_{1}^{2}+\cdots+n_{d}^{2},
\end{aligned}
$$

and the conditions (7.1) clearly hold. If $Q=I$, the identity operator, in $L^{2}(\mathcal{O})$, then Eq. 7.3 holds if and only if

$$
\delta<1-\frac{d}{2}
$$

Moreover, if $d=1$, Eq. 7.14 becomes $\delta<1 / 2$. If $d>1$, there is not such a value $\delta \in(0,1)$ satisfying Eq. 7.14.

For example, consider the following stochastic delay partial differential equation

$$
\left\{\begin{array}{l}
d y(t, \xi)=\frac{\partial^{2} y(t, \xi)}{\partial \xi^{2}} d t+\int_{-r}^{0} a(\theta) \frac{\partial^{2} y(t+\theta, \xi)}{\partial \xi^{2}} d \theta d t+d w(t, \xi), \quad t \geq 0, \quad \xi \in \mathcal{O}=[0, \pi]^{d}, \\
y(0, \cdot)=0, \quad y(\theta, \cdot)=0, \theta \in[-r, 0] \\
y(t, \xi)=0, \quad \xi \in \partial \mathcal{O}, \quad t \in(0, \infty),
\end{array}\right.
$$

where $r>0, a(\cdot)$ is some Hölder continuous function on $[-r, 0]$ and $w(\cdot, \cdot)$ is a standard Wiener random field on $\mathbb{R}_{+} \times \mathcal{O}$.

When $d=1$, we have in this case $\lambda_{k}=1, \mu_{k}=k^{2}, k \in \mathbb{N}$, and Eq. 7.3 holds for $\delta \in(0,1 / 2)$. The mild solution $y(t, \xi), t \geq 0, \xi \in[0, \pi]$, is thus $\alpha$-Hölder continuous with $\alpha \in(0,1 / 4)$.

Acknowledgements This work is supported by Project of Tianjin Municipal Education Commission (Grant No. JW1714).

Open Access This article is licensed under a Creative Commons Attribution 4.0 International License, which permits use, sharing, adaptation, distribution and reproduction in any medium or format, as long as you give appropriate credit to the original author(s) and the source, provide a link to the Creative Commons licence, and indicate if changes were made. The images or other third party material in this article are included in the article's Creative Commons licence, unless indicated otherwise in a credit line to the material. If material is not included in the article's Creative Commons licence and your intended use is not permitted by statutory regulation or exceeds the permitted use, you will need to obtain permission directly from the copyright holder. To view a copy of this licence, visit http://creativecommonshorg/licenses/by/4.0/.

\section{References}

1. Coleman, B.D., Gurtin, M.E.: Equipresence and constitutive equations for rigid heat conductors. Z. Angew. Math. Phys. 18, 199-208 (1967)

2. Da Prato, G., Zabczyk, J.: Stochastic Equations in Infinite Dimensions, 2nd edn. Encyclopedia of Mathematics and its Applications. Cambridge University Press, Cambridge (2014)

3. Di Blasio, G., Kunisch, K., Sinestrari, E.: $L^{2}$-regularity for parabolic partial integrodifferential equations with delay in the highest-order derivatives. J. Math. Anal Appl. 102, 38-57 (1984) 
4. Jeong, J.: Stabilizability of retarded functional differential equation in Hilbert space. Osaka J. Math. 28, 347-365 (1991)

5. Jeong, J., Nakagiri, S.I., Tanabe, H.: Structural operators and semigroups associated with functional differential equations in Hilbert spaces. Osaka J. Math. 30, 365-395 (1993)

6. Liu, K.: On regularity of stochastic convolutions of functional linear differential equations with memory. Discrete Contin. Dyn. Syst. Ser. B 25(4), 1279-1298 (2020)

7. Nunziato, J.W.: On heat conduction in materials with memory. Q. Appl. Math. 29, 187-204 (1971)

8. Tanabe, H.: On fundamental solution of differential equation with time delay in Banach space. Proc. Jpn. Acad. 64, 131-180 (1988)

9. Tanabe, H.: Equations of Evolution Monographs and Studies in Mathematics, vol. 6. Pitman, London (1979)

Publisher's Note Springer Nature remains neutral with regard to jurisdictional claims in published maps and institutional affiliations. 